\title{
Investigating Possible Boundaries Between Convergence and Divergence
}

\author{
Frederick Hartmann (frederick.hartmann@villanova.edu) and David Sprows \\ (david.sprows@villanova.edu), Villanova University, Villanova, PA 19085-1699
}

In the following, we describe a classroom discussion that can be used to supplement the material on the integral test for convergence and divergence of series.

One of the first applications of the integral test is to show that the series $\sum \frac{1}{n^{p}}$ converges for $p>1$ and diverges for $p \leq 1$ [Throughout this note, we assume that $p$ is a positive real number, and we omit the indices of infinite series because we are only concerned with the "tails" of these series.] Recently one of our students remarked that the harmonic series $\sum \frac{1}{n}$ acts like a boundary between convergence and divergence. Since multiplying $n$ in the denominator by a positive power of $n$ (no matter how small) produced a convergent series, this student conjectured that any series whose denominator involves $n$ multiplied by an expression that is always greater than 1 must be convergent. In other words, any series of positive terms which are term by term less than $\frac{1}{n}$ must converge. After it was pointed out that multiplication by a constant does not change convergence or divergence, this conjecture was quickly modified to what amounted to the following:

$$
\text { If } a_{n} \rightarrow \infty \text { as } n \rightarrow \infty \text {, then } \sum \frac{1}{a_{n} \cdot n} \text { converges }
$$

Since the unit considered in class involved the integral test, it was natural to investigate this conjecture by considering the case where $a_{n}=g(n)$ for some continuous function $g$. After a few false starts, the function $g(x)=\ln (x)$ was shown to produce a counterexample to (1). In fact, $\sum \frac{1}{n(\ln (n))^{p}}$ diverges for all $p \leq 1$. Since this series converges for $p>1$, it was natural to consider whether $n$ can be replaced by $n \ln (n)$ in (1). 
This new conjecture fails as can be seen by letting $a_{n}=\ln (\ln (n))$ and applying the integral test to $\sum \frac{1}{n \cdot \ln (n) \cdot \ln (\ln (n))}$. This pattern can be continued indefinitely. Let:

$$
\begin{gathered}
f^{0}(x)=x, f^{1}(x)=\ln (x)=f^{0}(\ln (x)), f^{2}(x)=\ln (\ln (x))=f^{1}(\ln (x)) \\
\text { and } f^{k+1}(x)=f^{k}(\ln (x)) k \geq 1 .
\end{gathered}
$$

Since the derivative of $f^{k}(x)$ equals $\frac{1}{f^{0}(x) \cdot f^{1}(x) \cdots f^{k-1}(x)}$, it follows by the integral test that $\sum \frac{1}{f^{0}(n) \cdot f^{1}(n) \cdots f^{k-1}(n) \cdot\left(f^{k}(n)\right)^{p}}$ converges for $p>1$ and diverges for $p \leq 1$. Once again, $p=1$ seems to be a dividing value between convergence and divergence. However, statement (1) still fails to be true when $\mathrm{n}$ is replaced by $f^{0}(n) \cdot f^{1}(n) \cdots f^{k}(n)$, since $a_{n}=f^{k+1}(n)$ gives the divergent series

$$
\sum \frac{1}{f^{k+1}(n) \cdot f^{0}(n) \cdot f^{1}(n) \cdots f^{k}(n)}=\sum \frac{1}{f^{k+1}(n)} D f^{k+1}(n) .
$$

Thus, the divergent series $\sum \frac{1}{f^{0}(n) \cdot f^{1}(n) \cdots f^{k+1}(n)}$ "slips" between the divergent series $\sum \frac{1}{f^{0}(n) \cdot f^{1}(n) \cdots f^{k}(n)}$ and the convergent series $\sum \frac{1}{f^{0}(n) \cdot f^{1}(n) \cdots f^{k-1}(n) \cdot\left[f^{k}(n)\right]^{p}}$ for $p>1$.

The above example shows that we can not use this approach to produce a boundary between divergence and convergence. The question arises: Will any procedure produce a "smallest" divergent series? The answer to this question is no, as can be seen from the following theorem in $[\mathbf{1}]$.

Theorem. If $\sum a_{n}$ is a divergent series of positive reals, then there exists a sequence $\epsilon_{1}, \epsilon_{2}, \ldots$ of positive numbers that converge to zero, but for which $\sum \epsilon_{n} \cdot a_{n}$ still diverges. 
Proof: Let $s_{n}=a_{1}+a_{2}+\cdots+a_{n}$. We first show $\sum_{k=1}^{\infty}\left(s_{k+1}-s_{k}\right) / s_{k+1}$ diverges. For any $m \in \mathbb{N}$ choose $n \in \mathbb{N}$ such that $s_{n+1}>2 s_{m}$. Since $\left\{s_{k}\right\}_{k}^{\infty}$ is non-decreasing,

$$
\begin{aligned}
\sum_{k=m}^{n} \frac{s_{k+1}-s_{k}}{s_{k+1}} & \geq \sum_{k=m}^{n} \frac{s_{k+1}-s_{k}}{s_{n+1}} \\
& =\frac{1}{s_{n+1}}\left[\left(s_{m+1}-s_{m}\right)+\left(s_{m+2}-s_{m+1}\right)+\cdots+\left(s_{n+1}-s_{n}\right)\right] \\
& =\frac{s_{n+1}-s_{m}}{s_{n+1}} \\
& >\frac{s_{n+1}-\frac{1}{2} s_{n+1}}{s_{n+1}}=\frac{1}{2} .
\end{aligned}
$$

Thus, the partial sums of the series $\sum_{k=1}^{\infty}\left(s_{k+1}-s_{k}\right) / s_{k+1}$ do not form a Cauchy sequence and so $\sum_{k=1}^{\infty}\left(s_{k+1}-s_{k}\right) / s_{k+1}=\infty$. Since $s_{k+1}-s_{k}=a_{k+1}$,

$$
\sum_{k=1}^{\infty} \frac{s_{k+1}-s_{k}}{s_{k+1}}=\sum_{k=2}^{\infty} \frac{a_{k}}{s_{k}} .
$$

Now let $\epsilon_{k}=1 / s_{k}$. Then $\epsilon_{k} \rightarrow 0$ and $\sum_{k=2}^{\infty} \epsilon_{k} a_{k}=\infty$.

It is worthwhile to make students realize that there is no specific series that can be used to establish a boundary between the set of all divergent positive-termed series and the set of all convergent series. Readers interested in a more detailed historical development of this topic may wish to consult [2].

References

1. Goldberg, R. R., Methods of Real Analysis, 2nd. edition, John Wiley \& Sons, New York, New York (1976) p. 71-72.

2. Knopp, K., Theory and Application of Infinite Series, Hafner Publishing, New York, New York (1947) P. 290-291. 